\title{
TeenSTAR: U na opción de madurez y libertad. Programa de educación integral de la sexualidad, orientado a adolescentes
}

\author{
Pilar Vigil $\mathbf{P}^{\mathbf{1}}$, Rosa Riquelme $\mathbf{R}^{\mathbf{1}, \mathbf{a}}$, Rosario Rivadeneira $\mathbf{H}^{\mathbf{1}}$, \\ W aldo Aranda ${ }^{2 b}$.
}
Effects of TeenSTAR, an abstinence only sexual education program, on adolescent sexual behavior

Background: Urgent measures are required to stop the increase in the frequency of pregnancies and sexually transmitted diseases among teenagers. A means of facing this problem is promoting sexual abstinence among youngsters. There are studies that confirm the efficacy of this approach. Aim: To show the results of the application of a holistic sexuality program (TeenSTAR) among Chilean teenagers. Subjects and Methods: Students attending basic or high school were divided into a control or study group. The control group (342 students) received the usual education on sexuality given by their schools and the study group (398 students) participated in twelve TeenSTAR sessions lasting 1.5 hours each, given by a trained professor. Assessment of achievements was made using an anonymous questionnaire answered at the start and end of the program. Results: The rates of sexual initiation among control and study groups were 15 and 6.5\%, respectively. Among sexually active students, $20 \%$ of those in the study group and 9\% of those in the control group discontinued sexual activity. Conclusions: A higher proportion of students in the TeenSTAR program retarded their sexual initiation or discontinued sexual activity and found more reasons to maintain sexual abstinence than control students (Rev Méd Chile 2005; 133: 1173-82).

(Key Words: Adolescent; Sexual abstinence; Sexual behavior)

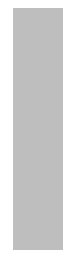

\footnotetext{
Recibido el 30 de julio, 2004. Aceptado el 24 de mayo, 2005.

${ }^{1}$ Unidad de Reproducción y Desarrollo, Departamento de Ciencias Fisiológicas, Facultad de Ciencias Biológicas, Pontificia Universidad Católica de Chile. ${ }^{2}$ Departamento de Estadística y Demografía, Escuela de Salud Pública, Facultad de Medicina, Universidad de Chile. Santiago de Chile.

aProfesora de Biología

bioestadístico.
}

Correspondencia a: Dra. Pilar Vigil P. Fax: 56-2-

2225515. E mail: pvigil@bio.puc.cl 
$\mathrm{E}^{1}$ inicio de la actividad sexual en los jóvenes chilenos es a los 12 años para los hombres y 14 años para las mujeres. Los estudios han demostrado que existe una tendencia a iniciar cada vez más temprano la actividad sexual. Estudios efectuados en Chile entre los años 1992 y 1995, mostraron que más de un tercio de los jóvenes inicia su actividad sexual antes del matrimonio y que la edad de inicio de la actividad sexual, entendida ésta como la primera relación sexual, era en promedio, entre los 14,4 y 16 años en los hombres y entre los 14,8 y 17,9 años en las mujeres ${ }^{1-5}$. Trabajos más recientes indican un inicio más precoz de la actividad sexual siendo de 12 años para los hombres y 12,8 años para las mujeres ${ }^{6}$.

La información existente en los adolescentes de enseñanza media acerca del uso de métodos anticonceptivos, muestra que $90 \%$ de los jóvenes conoce los diferentes métodos existentes para prevención de embarazo ${ }^{7}$. Sin embargo, su uso a esta edad es entre 27 y $45 \%$, siendo mayor en las mujeres que en los hombres $2,5,6,8$.

Además, existe información de que en nuestro país, durante 1999, 20,8\% del total de nacidos vivos era de madres menores de 20 años $^{9}$. Anualmente, en Chile, más de 50 mil adolescentes se convierten en madres. Estos datos no consideran los abortos ilegales, ni los embarazos que se inician a los 19 y terminan a los 20 años ${ }^{10,11}$.

Los datos expuestos anteriormente nos revelan la importancia de aplicar programas de educación integrales, que además de considerar la fisiología reproductiva, integren aportes para el desarrollo de la afectividad y la autoestima de los jóvenes y les enseñen técnicas que les permitan hacer frente a las presiones que sufren por parte de la sociedad en el ámbito sexual.

Los primeros programas de prevención de embarazo adolescente surgiemon en la década 1970-79 y se basaron en aumentar el conocimiento de los jóvenes acerca de las relaciones sexuales y de los riesgos y consecuencias del embarazo adolescente. La segunda generación de programas incluía la misma información, pero le daba mayor énfasis a la aclaración de valores y al desarmollo de técnicas de comunicación y toma de decisiones. La evaluación de todos los programas mencionados anteriormente no logró demostrar una disminución de la incidencia de conductas de riesgo sexual en adolescentes.

En respuesta a estos resultados, surgió una tercera generación de programas, que promovían la abstinencia sexual y excluían de sus cumículos toda información acerca de los métodos contraceptivos. Estos programas tampoco mostraron ser efectivos en la prevención de embarazo en adolescentes.

Finalmente, surgieron los programas de cuarta generación, que son una sumatoria de todos los anteriores. Enfatizan la prevención de enfermedades de transmisión sexual, incluida la inmunodeficiencia humana adquirida (VIH), combinando un fuerte mensaje pro abstinencia, con un entrenamiento en técnicas de comunicación y negociación. Al mismo tiempo, enseñan sexualidad y contracepción ${ }^{12}$.

Conscientes de que los padres son los primeros y principales educadores de sus hijos y que, en este campo, tienen una competencia fundamental, hemos implementado un programa de sexualidad orientado a ayudar en esta misión educativa.

El programa TeenSTAR se inició en Estados Unidos de Norteamérica en 1980, como un programa de educación en la sexualidad humana basado en el reconocimiento de la fertilidad cíclica, en el caso de las mujeres, y constante en el caso de los hombres, y en el descubrimiento de los valores inherentes a la posesión de esta sexualidad y fertilidad, que derivan en normas de comportamiento basadas en el entendimiento y respeto de ellas. Actualmente el programa se aplica en aproximadamente 35 países.

El presente estudio tuvo por objetivo evaluar el efecto del programa de sexualidad holístico TeenSTAR en la actividad sexual de adolescentes, aplicado durante un año académico, a jóvenes chilenos entre 12 y 18 años. Entendemos por actividad sexual a las relaciones sexuales coitales entre un hombre y una mujer. Se evaluaron específicamente tres aspectos principales: a) tasa de actividad sexual; b) tasa de discontinuación de actividad sexual y c) número de razones para mantener la abstinencia sexual.

\section{MATERIALES Y MÉTODOS}

a) Capacitación de profesores y aplicación del programa a jóvenes. El estudio abarcó a 934 estudiantes de enseñanza básica y media de 10 establecimientos educacionales de Chile. Para cuidar la diversidad, se escogieron en forma equivalente, colegios de diferente nivel socioeconómico y localización (Santiago y regiones), laicos 
y religiosos, de hombres, mujeres y mixtos. Participaron adolescentes, padres y educadores.

El programa TeenSTAR es un programa interactivo de educación holística en sexualidad humana, que se basa en la dignidad de la persona y en el uso de la libertad personal para la toma de decisiones. Permite integrar la capacidad biológica de ser padre o madre en todos los aspectos de la vida: social, emocional, intelectual, espiritual y físico. El programa pretende llevar al joven a efectuar sus propios descubrimientos, obtener conclusiones y tomar decisiones propias respecto de su comportamiento sexual. Ayudar a los jóvenes a vivir la experiencia de recibir y entender el mensaje que les envía su cuerpo respecto de su fertilidad y de su capacidad recién adquirida de procrear, da al joven un aprendizaje no sólo intelectual, sino que le permite tomar una decisión libre frente a la expresión de su comportamiento sexual.

Dentro de los objetivos del programa TeenSTAR está el fortalecimiento de las siguientes áreas: a. Fortalecer la identidad propia y mejorar la autoestima. Los jóvenes necesitan saber quiénes son. Se les invita a tomar conciencia que son personas libres, pero limitadas.

b. Valorar su libertad y su capacidad de decisión. Se informa a los jóvenes acerca de su libertad y responsabilidad respecto de sus opciones.

c. Desarrollar en los jóvenes el respeto por el don de la vida. La vida humana es un don recibido para ser, a su vez, donado. Sólo aquellos jóvenes que valoren su propia vida podrán entregarla como un don a otras personas. $\mathrm{Si}$ el joven se desprecia a sí mismo, despreciará la vida y no verá en su posible entrega un valiosísimo don.

La metodología empleada en el estudio comprende la capacitación de profesores, en un seminario-taller de 40 horas cronológicas. La población del estudio abarcó a alumnos de enseñanza básica y media, entre 12 y 18 años. Los jóvenes se dividieron en Jóvenes Programa, correspondiente a los jóvenes con los cuales se trabajó el programa de educación sexual TeenSTAR, y Jóvenes Control.

El trabajo con los jóvenes del Programa TeenSTAR abarcó un año académico, se consideró un cuméculo diferenciado según edad y sexo, aplicándose un curńculo para cada nivel de edad. Como parte del programa, el monitor efectuó por lo menos una entrevista personal con cada alumno. El programa se desamolló con el consentimiento conjunto de padres y adolescentes. Sólo $2 \%$ de los alumnos a los que fue ofrecida esta posibilidad de formación decidió no asistir y, por lo tanto, no se incluyeron en el estudio.

El programa se aplicó de forma aleatoria, prospectiva y como un ensayo controlado que comparó una intervención basada en la abstinencia sexual con la educación normalmente impartida por los colegios.

El estudio fue aprobado por el consejo de dirección de todos los establecimientos educacionales participantes y por el comité de ética de la Facultad de Ciencias Biológicas de la Pontificia Universidad Católica de Chile.

Se incluyó en el estudio a todos los alumnos que desearon participar y que tenían el consentimiento de sus padres. Se excluyeron, previamente al inicio del estudio, a todos los jóvenes menores de 12 y mayores de 18 años (ambos extremos de la curva de distribución normal) y, para el análisis de resultados, a todos los estudiantes cuyos pre y post test no fueran concordantes en algún dato, como fecha de nacimiento, y por la presencia de datos poco confiables, es decir, cuestionarios sin correlación entre la fecha de nacimiento y el código de identificación, omisión de preguntas 0 no correlación sobre respuestas a preguntas doblemente realizadas en el cuestionario. Para determinadas variables, se consideró como criterio de exclusión la no respuesta o falta de concordancia en las respuestas de alguna pregunta específica.

Participaron un total de 934 adolescentes, los que se dividieron en Jóvenes Programa y Jóvenes Control en forma aleatoria. El grupo control (342 alumnos) recibió la educación normalmente impartida por el colegio al que asistía y el grupo programa (398 alumnos) recibió 12 sesiones de una hora y media cada una, separadas por 15 días, impartidas por un profesor capacitado en TeenSTAR.

Entre las limitaciones que tiene un estudio de estas características, está el que el programa se aplicó en colegios diferentes, cuyos programas educacionales pueden ser disímiles y pueden funcionar de formas distintas y a que las características propias de cada monitor pueden influir en los resultados, aunque todos hayan sido formados en el mismo taller. Con respecto a la evaluación, los alumnos podían omitir responder las preguntas que quisieran. Esto, sumado a la falta de concondancia de algunas respuestas entre el pre y el post test, llevó a 
que prefiriéramos dejar fuera del estudio a estos estudiantes (para evitar el sesgo) y explica que, para ciertos análisis, como la tasa de transición de actividad sexual, el número de estudiantes evaluados sea menor al tamaño muestral inicial. Además, hay que considerar que un año de observación de la muestra es insuficiente para establecer pronósticos a largo plazo en esta materia.

b) Evaluación. La evaluación de los logros del programa se realizó a través de un cuestionario anónimo, cerrado, que fue contestado por los jóvenes programa y control, en tiempos paralelos, durante la sesión inicial y final del programa. El cuestionario consistió en 135 preguntas que abarcaban diferentes tópicos, en los cuales se incluía la ocurrencia de actividad sexual. A final de año, se correlacionaron las respuestas de los cuestionarios de inicio y término, tanto en el grupo control como programa. Del total de 934 adolescentes que participaron en el estudio, se consideraron como válidos 79,2\% de los cuestionarios. Se excluyó a $11,5 \%$ por ausentismo al tomar el cuestionario inicial o final y 9,3\% se excluyó por omisión de variables fundamentales para el análisis computarizado, como fecha de nacimiento, y por la presencia de datos poco confiables, es decir, cuestionarios sin correlación entre la fecha de nacimiento y el código de identificación, omisión de preguntas 0 falta de correlación sobre respuestas a preguntas doblemente realizadas en el cuestionario. La tasa de exclusión del estudio por estas causas no tuvo diferencias entre los grupos programa y control.
Para el análisis estadístico de los resultados, se utilizó la prueba de t de Student para determinar la existencia de diferencias significativas entre ambos grupos. El cuestionario utilizado fue confeccionado y validado por el doctor Stan Weed en el institute of Research and Evaluation», en Salt Lake City, Estados Unidos $^{13-16}$. Se utilizaron dos pruebas estadísticas de homogeneidad para confirmar que la tasa de respuestas obtenidas en el grupo programa, con la tasa de respuestas obtenidas en el grupo control son homogéneas (test de homogeneidad $p>0,05$ ).

\section{Resultados}

Al final del desarrollo del programa se obtuvo la evaluación de 740 jóvenes, 446 hombres y 294 mujeres. Los grupos diferenciados por edad y sexo se aprecian en la Tabla 1.

Las variables demográficas mostradas en la Tabla 2 indican que 76,8\% de los jóvenes estudiados vivía con sus padres. En relación al nivel educacional de éstos últimos, tenían educación media completa o educación superior $21,2 \%$ de las madres y $31,2 \%$ de los padres. El $81,1 \%$ de los jóvenes se reconoció como católico.

A continuación, se analizarán en forma separada los resultados obtenidos en relación a la tasa de actividad sexual (definida como el haber tenido relaciones sexuales coitales en los últimos 3 meses), discontinuación de actividad sexual y número de razones que tienen los jóvenes para mantener la abstinencia.

Tabla 1. Adolescentes encuestados según edad y sexo

\begin{tabular}{|lcccc|}
\hline Edad (años) & Sexo & Programa (n) & Control (n) & Total (n) \\
\hline \multirow{2}{*}{$12-14$} & $\mathrm{H}$ & 110 & 74 & 184 \\
& $\mathrm{M}$ & 12 & 0 & 12 \\
$15-16$ & $\mathrm{H}$ & 96 & 69 & 165 \\
& $\mathrm{M}$ & 47 & 75 & 122 \\
$17-18$ & $\mathrm{H}$ & 45 & 52 & 97 \\
& $\mathrm{M}$ & 88 & 72 & 160 \\
Total/sexo & $\mathrm{H}$ & 251 & 195 & 446 \\
\multirow{2}{*}{ Total } & $\mathrm{M}$ & 147 & 147 & 294 \\
& & 398 & 342 & 740 \\
\hline
\end{tabular}

${ }^{*} \mathrm{p}>0,2$ 
a) Tasa de actividad sexual. El presente estudio mostró que $20 \%$ de los adolescentes entre 12 y 18 años ya ha tenido, al menos, una relación sexual (Tabla 2), siendo mayor esta actividad en hombres que en mujeres (22,9\% y 15,7\%, respectivamente).
En todos los rangos de edad evaluados se observó un mayor porcentaje de hombres que mujeres con actividad sexual (Tablas 3, Tabla 4 y Figura 1).

La mayoría de los jóvenes encuestados (86,5\%) respondieron que su primera relación sexual fue

Tabla 2. Variables socio-demográficas y conducta sexual del grupo estudiado

\begin{tabular}{|c|c|c|c|c|c|}
\hline \multirow[t]{2}{*}{ Variable } & \multicolumn{2}{|c|}{$\begin{array}{c}\text { Programa } \\
398\end{array}$} & \multicolumn{2}{|c|}{$\begin{array}{c}\text { Control } \\
342\end{array}$} & \multirow{2}{*}{$\begin{array}{c}\text { Total } \\
740 \\
\%\end{array}$} \\
\hline & $\mathrm{n}$ & $\%$ & $\mathrm{n}$ & $\%$ & \\
\hline Promedio edad (años) & \multicolumn{2}{|c|}{15,2} & \multicolumn{2}{|c|}{15,6} & \\
\hline Vive con sus padres & 270 & 67,8 & 299 & 82,6 & 76,8 \\
\hline Educación madre (media completa o superior) & 78 & 19,6 & 79 & 21,8 & 21,2 \\
\hline Educación padre (media completa o superior) & 117 & 29,4 & 114 & 31,5 & 31,2 \\
\hline Religión católica & 287 & 72,1 & 313 & 86,5 & 81,1 \\
\hline Con actividad sexual & 75 & 18,8 & 73 & 21,3 & 20,0 \\
\hline Promedio edad primera relación sexual (años) & \multicolumn{2}{|c|}{14,3} & \multicolumn{2}{|c|}{14,8} & 14,6 \\
\hline Promedio edad primera pareja (años) & \multicolumn{2}{|c|}{17,9} & \multicolumn{2}{|c|}{18,7} & 18,3 \\
\hline Circunstancias: consentimiento mutuo & 69 & 92 & 59 & 80,8 & 86,5 \\
\hline \multicolumn{6}{|l|}{ Trató de prevenir embarazo: } \\
\hline Siempre & 22 & 29,3 & 18 & 24,7 & 27,0 \\
\hline La mayoría de las veces & 12 & 16,0 & 15 & 20,5 & 18,2 \\
\hline Sabe de técnicas para prevenir embarazo & 68 & 90,7 & 62 & 84,9 & 87,8 \\
\hline
\end{tabular}

${ }^{*} \mathrm{p}>0,1$.

Tabla 3. Jóvenes con actividad sexual al inicio del estudio, distribuidos por edad

\begin{tabular}{|llrrrrrr|}
\hline \multirow{2}{*}{ Edad } & Sexo & \multicolumn{2}{c}{ Jóvenes } & \multicolumn{2}{c}{ Jóvenes } & \multicolumn{2}{c|}{ Total } \\
& & \multicolumn{2}{c}{ Programa } & \multicolumn{2}{c}{ Control } & \multicolumn{2}{c|}{ Grupo } \\
& & $\mathrm{n}$ & $\%$ & $\mathrm{n}$ & $\%$ & $\mathrm{n}$ & $\%$ \\
\hline \multirow{2}{*}{$12-14$} & $\mathrm{H}$ & 12 & 10,9 & 12 & 16,2 & 24 & 13,0 \\
& $\mathrm{M}$ & 1 & 8,3 & 0 & 0,0 & 1 & 8,3 \\
$15-16$ & $\mathrm{H}$ & 25 & 26,0 & 16 & 23,2 & 41 & 24,8 \\
& $\mathrm{M}$ & 6 & 12,8 & 10 & 13,3 & 16 & 13,1 \\
$17-18$ & $\mathrm{H}$ & 15 & 33,3 & 22 & 42,3 & 37 & 38,1 \\
& $\mathrm{M}$ & 16 & 18,2 & 13 & 18,1 & 29 & 18,1 \\
Total & $\mathrm{H}$ & 52 & 20,7 & 50 & 25,6 & 102 & 22,9 \\
& $\mathrm{M}$ & 23 & 15,6 & 23 & 15,6 & 46 & 15,7 \\
\hline
\end{tabular}

$*_{p}>0,3$ 
Tabla 4. Tasa de actividad sexual distribuida por sexo

\begin{tabular}{|lcc|}
\hline & $\begin{array}{c}\text { Hombres } \\
(446)\end{array}$ & $\begin{array}{c}\text { Mujeres } \\
(294)\end{array}$ \\
\hline Edad promedio de la primera relación sexual (años) & 13,9 & 15,5 \\
Promedio edad de la pareja (años) & 17,6 & 19,3 \\
Jóvenes con actividad sexual (\%) & 22,9 & 15,7 \\
\hline
\end{tabular}

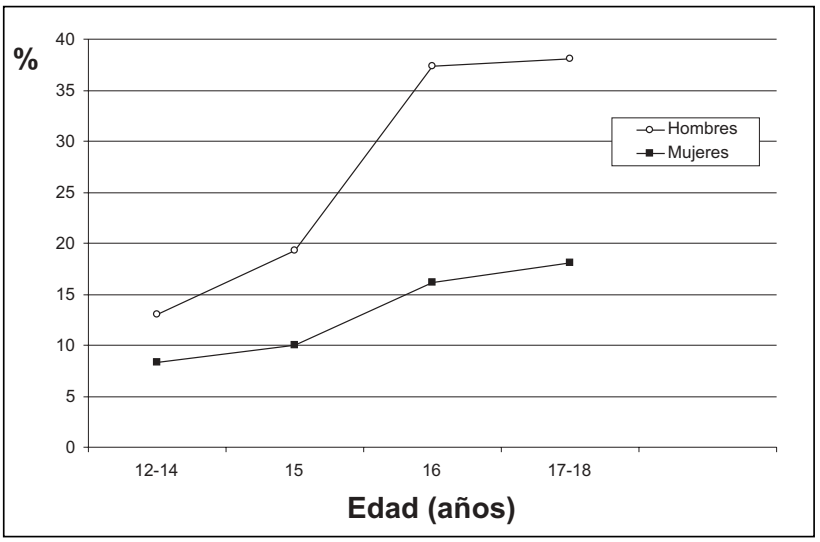

FiguRA 1. Relaciones sexuales en jóvenes distribuidos por edad y sexo.

por consentimiento mutuo, es decir, tuvieron una relación sexual porque así «o desearon». La primera relación sexual en los hombres ocurrió más precozmente que en las mujeres, en promedio a los 13,9 años en los hombres y 15,5 años en las mujeres. Se observó, en ambos casos, que las parejas sexuales involucradas en esta primera relación eran de mayor edad y tenían, en promedio, 17,6 y 19,3 años para varones y mujeres, respectivamente (Tabla 4).

Entre los 12 y 14 años, 13,4\% de los jóvenes de sexo masculino ha tenido, al menos, una relación sexual. Este porcentaje aumenta hasta llegar a $38,1 \%$ a los 17-18 años. Si bien las mujeres inician su actividad sexual más tardíamente que los hombres, se observa un importante aumento durante el período de término de la enseñanza media (17-18 años), llegando a una tasa de actividad sexual de 18,1\% (Tabla 3 y Figura 1).

Es importante destacar el hecho de que los datos observados previamente a la aplicación del programa en los diferentes establecimientos estudiados, no mostraron diferencias significativas entre sí y que tampoco existieron diferencias significativas entre los jóvenes programa y control $(\mathrm{p}>0,1)$.
Al ser consultados sobre el uso de métodos anticonceptivos, $27 \%$ de los alumnos indicó que «siempre» trató de prevenir el embarazo por esta vía y $18,2 \%$ lo hizo «a mayoría de las veces». Esto, a pesar de que $87,8 \%$ de los jóvenes sexualmente activos, indicó conocer las técnicas que existen para prevenir el embarazo (Tabla 2).

Al realizar el análisis del efecto del programa TeenSTAR en los jóvenes participantes (Figuras 2 y 3), se observó que la tasa de iniciación de vida sexual, es decir, la transición de virgen a no virgen fue de $15,3 \%$ por año en el grupo control versus $6,5 \%$ en los jóvenes participantes del programa (Figura 2). Al realizar el análisis por sexo, se observó que la tasa de iniciación para los hombres fue de $17,6 \%$ versus $8,8 \%$ en jóvenes control y programa, respectivamente ( $\mathrm{p}<0,004)$; y para las mujeres fue de $12,4 \%$ en jóvenes control y $3,4 \%$ en jóvenes programa, respectivamente ( $p<0,0001$ ) (Figura 3). El tamaño de la muestra en estos casos fue menor al inicial (492 vs 740 ) ya que se incluyeron los alumnos que cumplieron con los criterios de haber contestado la pregunta de conducta sexual en ambos test y que las respuestas fueran concordantes, además de los criterios de inclusión analizados antes en este trabajo. 
En el transcurso del estudio hubo que lamentar pérdidas de unidades pertenecientes a la muestra, tanto del grupo que pertenecía al programa como del grupo control. Con el fin de comprobar que la pérdida de unidades muestrales afectó por igual a ambos grupos en el estudio, se realizaron dos pruebas estadísticas que confirmaron, que la tasa de respuestas obtenidas en el grupo dentro del programa, con la tasa de respuestas obtenidas fuera del programa, eran homogéneas (test de homoge- neidad $p>0,05)$. Esto quiere decir que la proporción de personas que terminó el estudio en el grupo del programa, no difiere significativamente de la proporción de personas que terminó el estudio y no pertenecía al programa. La misma conclusión se obtuvo haciendo una prueba Z, basada en la distribución normal en que se compara la tasa que termina el estudio en el grupo del programa con la tasa que termina el estudio en el grupo fuera de programa (Tabla 5).

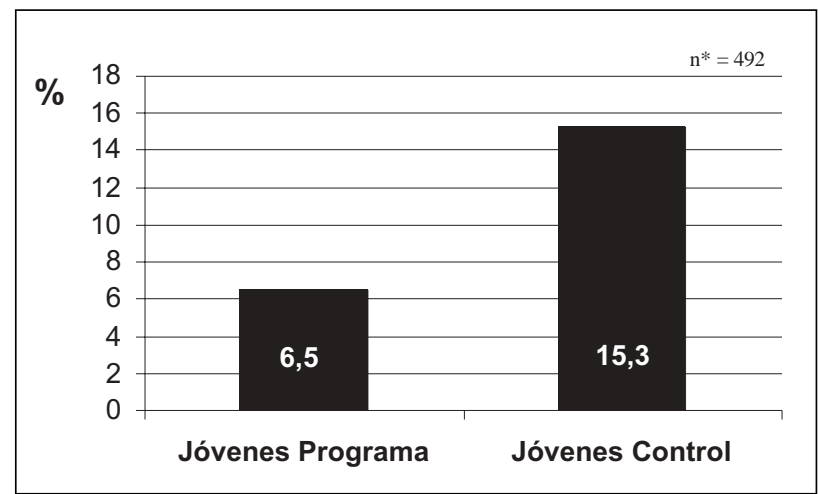

FigURA 2. Jóvenes que inician su actividad sexual durante el año de estudio.

$\mathrm{p}<0,05$.

${ }^{*}$ Representa el número total de jóvenes que contestaron esta pregunta.

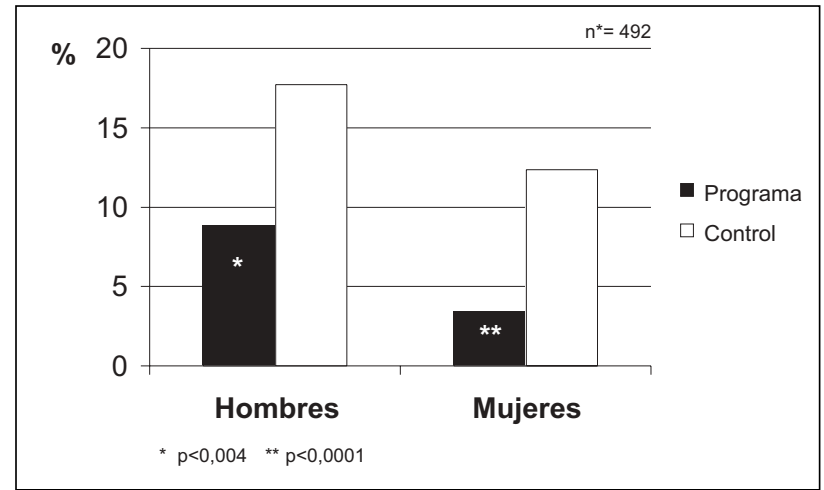

Figura 3. Tasa de transición de actividad sexual de jóvenes programa y control según sexo.

n: Representa el número total de jóvenes que contestaron esta pregunta.

Tabla 5. Pérdida de unidades muestrales en relación a la pregunta de actividad sexual. Comparación entre los grupos Programa y Control

\begin{tabular}{|lccc|}
\hline & Grupo & Grupo & Total \\
& Programa & Control & \\
\hline Terminan el estudio & 276 & 216 & 492 \\
& $(69,35 \%)$ & $(63,16 \%)$ & \\
No terminan el estudio & 122 & 126 & 248 \\
& $(30,65 \%)$ & $(36,84 \%)$ & 740 \\
Total en estudio & 398 & 342 & \\
& $(100 \%)$ & $(100 \%)$ & \\
\hline
\end{tabular}


La distribución de los alumnos excluidos de los grupos programa y control no tuvo una diferencia estadísticamente significativa, por lo que la exclusión de dichos cuestionarios no afectaría la validez de los resultados del estudio.

b) Discontinuación de la actividad sexual. Veinte por ciento de los jóvenes incluidos en el programa ya habían iniciado su actividad sexual. Estos se dividieron en: a) los que se encontraban sexualmente activos al inicio del programa (es decir, que habían tenido, al menos, una relación sexual en los últimos tres meses), y b) los jóvenes que ya habían tenido una relación sexual, pero que por diversas razones habían discontinuado su actividad sexual (mayor o igual a tres meses de inactividad sexual). Los resultados mostraron que $20 \%$ de los jóvenes programa sexualmente activos al inicio de éste discontinuaron su actividad sexual después de la intervención. Ello, en comparación con $9 \%$ del grupo control $(p<0,03)$. Con respecto al grupo de jóvenes que ya habían tenido una relación sexual, pero que por diversas razones habían discontinuado su actividad sexual (mayor o igual a tres meses de inactividad sexual), el estudio mostró que $11,7 \%$ reinició su actividad sexual al final de la intervención, no observándose un reinicio de la actividad sexual en los jóvenes programa ( $p<0,04)$.

c) Aumento del número de razones para la abstinencia sexual. Al analizar las razones que tenían los jóvenes para no iniciar su actividad sexual (principios religiosos, miedo a embarazo y contagio de enfermedades de transmisión sexual, no haber encontrado a la persona adecuada, no sentirse preparado, no tener la oportunidad, temor de decepcionar a los padres, no querer ser usado, entre otras), observamos que, en los jóvenes programa, ocurrió un aumento del número de razones para no tener una relación sexual, pasando de tener sólo una razón al inicio del programa, a dos o tres razones al final de éste. En los jóvenes controles no hubo variación en este parámetro.

\section{DiscUSIÓN}

El aumento de los embarazos en adolescentes y el gran porcentaje de enfermedades de transmisión sexual que concentra esta población, hacen deseable el logro de cambios en la conducta sexual de los adolescentes. Una manera de abordarlo, que cada día gana más adeptos, es la promoción de la abstinencia sexual. El concepto de abstinencia sexual incluye tanto la abstinencia primaria, es decir, el retraso de la edad de iniciación sexual; como la abstinencia secundaria, entendida como la discontinuación de la actividad sexual cuando ésta ya ha sido iniciada.

La educación en la abstinencia sexual se define como la enseñanza de los beneficios de esta práctica en términos de ganancia en los ámbitos social y de salud, pero también de las posibles consecuencias de la actividad sexual en adolescentes como serían el riesgo de embarazo y contagio de enfermedades de transmisión sexual $^{12}$

La abstinencia es un método que provee total protección para evitar el embarazo adolescente y las enfermedades de transmisión sexual. Además, ningún estudio ha probado que su promoción sea deletérea para los jóvenes y no tiene efectos secundarios indeseados.

Algunos programas de abstinencia sugieren que los adolescentes no son capaces de comprender la totalidad de las implicancias de su actividad sexual y no son lo suficientemente maduros como para manejar las consecuencias de ésta. Por lo anterior, es importante que los programas de educación en esta área enseñen a saber decir que NO frente a las presiones sexuales»a las que se ven sometidos los adolescentes. Diversos estudios muestran que los adolescentes identifican a las presiones para tener relaciones sexuales como la primera amenaza a su salud y que lo que más desearían saber es scómo decirle que no a una pareja sin perderla» ${ }^{17,18}$. Por otra parte, una vez que los adolescentes comienzan a tener una vida sexual activa, tienden a continuarla en el tiempo. Es importante destacar que entre los logros del programa TeenSTAR está el aumento de la tasa de discontinuación de actividad sexual.

Algunas investigaciones han demostrado que los adolescentes están en una etapa de desarrollo, caracterizada por un procesamiento parcial de la información y por una falla en la capacidad de anticipar las consecuencias futuras de sus acciones ${ }^{19}$. Esto se conoce como la invulnerabilidad del adolescente y es lo que determinaría que, para 
ellos, sea difícil comprender y utilizar los métodos anticonceptivos de manera eficiente y consistente. Esto explicaría que, si bien la mayoría de los adolescentes conocen cuáles son y cómo se usan los diversos métodos anticonceptivos, no los utilizan $^{12}$. Con respecto a esto, nuestro estudio arroja un leve aumento en la tasa de uso de métodos anticonceptivos en las relaciones sexuales, de $13,5 \%$, presentada en trabajos previos, a $18,2 \% 3,20,21$. También se observó un aumento con respecto al conocimiento que se tiene de ellos, de $54,8 \%$ a $87,8 \%{ }^{10}$. Sin embargo, los datos obtenidos en este trabajo siguen siendo concordantes con la hipótesis de la invulnerabilidad.

Estudios previos, en que se han evaluado las diferencias de género en la sexualidad adolescente, han demostrado que las mujeres tienen mayor tendencia a la abstinencia sexual que los hombres. La causa de esto se atribuyó a que ellas percibirían tener mayor control que los hombres sobre sus impulsos sexuales ${ }^{14}$. Esto es concordante con los resultados de nuestro trabajo y es importante considerarlo al elaborar y al aplicar programas de educación sexual.

Es difícil comparar los programas de educación sexual entre sí porque no son del todo iguales y el énfasis que se da a diferentes áreas no es el mismo. Sin embargo, la evaluación de diferentes programas de educación sexual para adolescentes, ha permitido determinar ciertas características de éstos que hacen que logren promover la abstinencia sexual entre los adolescentes $^{19}$. Éstas son:

- Que estén fundados en teorías de conductas saludables (por ejemplo, promover la actividad física y evitar el tabaco y el alcohol).

- Que incluyan a los padres para lograr su mayor participación y para mejorar el nivel de comunicación con los adolescentes.

- Confrontar las influencias sociales y de los medios de comunicación con los valores familiares y con conductas saludables. Evaluar en qué difieren unas de otras.

- Entrenamiento en técnicas de negación consistentes y adecuadas a su edad y entorno social.

- Incluir el juego de roles, que permite practicar las técnicas de negación, negociación y comu- nicación. Les permite además imaginarse situaciones en las que podrían encontrarse.

- Utilizar material anexo como videos, historietas, ropa, posters, entre otros.

El programa de educación sexual para adolescentes TeenSTAR incluye todos los elementos de éxito mencionados anteriormente. Se basa esencialmente en lograr que los jóvenes conozcan y comprendan los mensajes que les envía su cuerpo en relación a su fertilidad, a la vez que les enseña a formarse y valorarse como personas íntegras, dignas de todo el respeto que ellos mismos, sus pares y la sociedad completa les deben. Se desarrollan los conceptos de intimidad, libertad y responsabilidad. Al mismo tiempo, se informa a los jóvenes sobre los distintos métodos anticonceptivos y las formas de prevenir las enfermedades de transmisión sexual. Nunca se les niega información, solamente se les educa para que puedan recibirla y utilizarla responsablemente.

Los programas de educación sexual tendientes a retrasar el inicio de la actividad sexual muestran ser efectivos ${ }^{4,23,25-27}$. De acuerdo a estos estudios, los programas que desarrollan la valoración por la persona, así como la decisión libre e informada, tendrían mayor impacto en los adolescentes.

El programa TeenSTAR demostró ser efectivo en retrasar la edad de iniciación sexual y también mostró resultados positivos en relación a la discontinuación de la actividad sexual y al número de razones que tienen los jóvenes para mantener la abstinencia sexual. Estos resultados se obtuvieron en todos los grupos estudiados, independiente de las variables demográficas expuestas previamente en este trabajo y de la disminución del tamaño muestral por la exclusión de cuestionarios considerados como no válidos.

Los programas de educación sexual de esta índole serían una opción válida para enfrentar el problema del embarazo adolescente y las enfermedades de transmisión sexual. Creemos que estudios de este tipo permitirán mantenernos informados acerca de las características del comportamiento sexual de nuestros adolescentes para poder así mejorar los programas de educación ya existentes. 


\section{REFERENCIAS}

1. Fuentes ME, Lobos L Adolescente embarazada. Ed. Universitaria. Santiago de Chile, 146 Pág, 1994.

2. MiLÁn T, ValenZueia S, Vargas NA. [Reproductive health in adolescent students: knowledge, attitudes and behavior in both sexes, in a community of Santiago]. Rev Méd Chile 1995; 123: 368-75.

3. Reposi A, Araneda JM, Bustos L, Puente C, Rojas C. [Sexual behaviors and contraceptive practices among university students]. Rev Méd Chile 1994; 122: 27-35.

4. SATHER L, ZinN K. Effects of abstinence-only education on adolescent attitudes and value concerning premarital sexual intercourse. Fam Community Health 2002; 25: 1-15.

5. Valenzuela MS, Herold JM, Morris L, López IM. [Reproductive health survey of young adults in Greater Santiago]. Bol Oficina Sanit Panam 1992; 112: 19-30.

6. Fernández L, Bustos L, González L, Palma D, ViLAGRÁN J, MuÑoz S. [Beliefs, attitudes and knowledge about sex education]. Rev Méd Chile 2000; 128: 571-3.

7. Molna R, Araya S, Ibazeta G, Jordán P, Lagos E. Nivel de conocimientos y práctica de sexualidad en adolescentes. Rev Chil Obstet Ginecol 1986; 3: 293-301.

8. Valenzuela MS, Herold JM, Lorris L, López IM. Encuesta de salud reproductiva en adultos jóvenes del Gran Santiago. Bol Oficina Sanit Panam 1992; 112 (1).

9. MINSAL Política nacional de salud para adolescentes y jóvenes. Santiago de Chile, 1999.

10. Álvarez M, Mauricci A, Muzzo S. Información sexual de los adolescentes según sexo. Rev Chil Pediatr 1990; 61: 102-8.

11. Delpiano A, Aguilera M. Mujeres Chilenas: Estadísticas para el Nuevo Siglo. Instituto Nacional de Estadística, 2001, páginas 25-28.

12. Thomas M. Abstinence Based Programs for Prevention of Adolescent Pregnancies. J Adolesc Health 2000; 26: 5-17.

13. De Gaston JF, Jensen L, WeEd S. A closer look at Adolescent Sexual Activity. Journal of Youth and Adolescence 1995; 24: 465-80.

14. De Gastón JF, WeEd S, Jensen L Understanding gender differences in adolescent sexuality. Adolescence 1996; 31: 217-31.
15. Olsen JA, WeEd SE. Effects of Family Planning Programs for Teenagers on Adolescent Birth and Pregnancy Rates. Family Perspective 1986; 20: 153-70.

16. Weed SE, Olsen JA. Effects of Family Planning Programs on Teenage pregnancy - Replication and Extension. Family perspective 1986; 20: 17394.

17. Kirby D, Barth RP, Leland N, Fetro JV. Reducing the risk: Impact of a new curriculum on sexual risk- taking. Fam Plann Perspect 1991; 39: 363-7.

18. Howard M, McCABE JB. Helping teenagers postpone sexual involvement. Fam Plann Perspect 1990; 22: 21-6.

19. Hall PA, Holmovist M, Sherry SB. Risk Adolescent Sexual Behavior: A Psychological Perspective for Primary Care Clinicians. Topics in Advance Practice Nursing Journal 2004 (1), Medscape.

20. Harold JM, Valenzuela MS, Morris L. Premarital sexual activity and contraceptive use in Santiago, Chile. Stud Fam Plann 1992; 23: 128-36.

21. Valenzueia MS, Herold JM, Morris L, López I. Encuesta de salud reproductiva en adultos jóvenes: Experiencia sexual y uso de anticonceptivos. Rev Méd Chile 1990; 118: 465-72.

22. Carter-Jessor L, Frankin LN, Heath JW, JimÉnEz-Irizarry G, PEACE MD. Abstinence education for urban youth. J Community Health 2000; 25: 293-304.

23. DarRoch JE, LandRY DJ, Singh S. Changing emphases in sexuality education in U.S. public secondary schools, 1998-1999. Fam Plann Perspect 2000; 32: 204-211, 265.

24. Doniger AS, Adams E, Utter CA, Ruey JS. Impact evaluation of the not, not now» abstinenceoriented adolescent pregnancy prevention communications program, Monroe County, New York. J Health Commun 2000; 6: 45-6.

25. Olsen JA, WeEd SE, Ritz GM, Jensen LC. The effects of three-abstinence sex education programs on student attitudes toward sexual activity. Adolescent 1991; 26: 631-41.

26. Roper Starch Worddwide. Teens Talk About Sex: Adolescent Sexuality in the 90's. New York. Sexuality Information and Education Council of the United States, 1994.

27. Kiaus H, Bryan L, Bryant M, Fagan M, Harrigan M, KeARNS F. Fertility awareness/natural family planning for adolescents and their families: Report of multisite pilot project. Int J Adol Med and Health 1987; 3: 101-19. 